\title{
Etude du transfert de quelques oligo-éléments dans les chaînes trophiques néritiques et estuariennes: Accumulation biologique chez les poissons omnivores et super-carnivores
}

\author{
C. Metayer ${ }^{1}$, J.-C. Amiard ${ }^{2}$, \\ C. Amiard-Triquet ${ }^{2}$ \& J. Marchand ${ }^{2 *}$ \\ 'Laboratoire de Chimie Analytique et d'Hydrologie, \\ $U$. E. R. des Sciences Pharmaceutiques; \\ 1, rue Gaston Veil, F-44035 - Nantes Cedex, France \\ ${ }^{2}$ Laboratoire de Biologie Marine, U. E. R. des Sciences de la Nature; \\ 2, rue de la Houssinière, F-44072 - Nantes Cedex, France
}

\begin{abstract}
On the transfer of several trace elements in neritic and estuarine food chains: Bioaccumulation in omnivorous and carnivorous fishes. Five species of fishes (Dicentrarchus labrax, Gobius microps, Stizostedion lucioperca, Gadus luscus, Merlangius merlangus) and their major prey organisms were collected monthly from two stations in the Loire estuary (France). The levels of several trace elements $(\mathrm{Cd}, \mathrm{Pb}, \mathrm{Cu}, \mathrm{Zn})$ in their tissues were determined by means of atomic absorption spectrophotometry. The concentrations of $\mathrm{Cd}, \mathrm{Pb}$ and $\mathrm{Cu}$ were shown to decrease in the highest trophic levels: the relatively highest metal levels were determined in annelids, followed by crustaceans, the lowest levels being encountered in fishes. However, a preferential uptake of $\mathrm{Cu}$ was observed in crustaceans. There is no biomagnification for these three metals, the concentrations in preys being generally lower than in predators. For $\mathrm{Zn}$, the highest concentrations were measured in worms and copepods but preys such as shrimps and mysids exhibit values of the same order of magnitude compared to predator fishes.
\end{abstract}

\section{INTRODUCTION}

Milieux très productifs (aux Etats-Unis, $70 \%$ des espèces commercialisées ont une partie de leur cycle biologique en estuaire d'après Rice et al. (1972), mais zones fortement industrialisées ou en voie d'industrialisation, les estuaires doivent être l'objet d'études approfondies dans une optique de protection de l'environnement et des richesses naturelles. Dans l'estuaire de la Loire, ce double aspect productivité-pollution a été abordé par l'analyse de la structure du réseau trophique (Marchand, 1978) et par une évaluation de l'accumulation biologique de certains métaux anthropogènes ou d'origine naturelle (Amiard et al., 1980). La répartition des métaux dans les réseaux alimentaires a ensuite été envisagée dans le cas simple de la chaîne alimentaire: producteurs primaires

- Avec la collaboration technique de R. Ferre. 
$\rightarrow$ zooplancton $\rightarrow$ Poissons planctonophages. Dans ce cas, l'accumulation biologique des métaux s'est révélée généralement beaucoup moins importante chez les Poissons que chez les Crustacés planctoniques qui constituent leurs proies. A part quelques exceptions concernant principalement le zinc, on peut dire qu'il n'a pas été observé de phénomène de biomagnification (Amiard-Triquet et al, 1980).

Nous envisageons maintenant la concentration des métaux ches des espèces présentant des régimes alimentaires complexes et chez quelques super-carnivores. Le Bar (Dicentrarchus labrax) est un Poisson marin qui pénètre dans les eaux saumâtres de l'estuaire surtout pendant la période estivale. Les jeunes individus que nous avons prélevés (1 à 2 ans) étaient susceptibles d'utiliser comme nourriture du zooplancton (Copépodes, Mysidacées), des petits Crustacés benthiques vagiles (Crangon crangon) mais surtout de la faune endogée (Annélides: Boccardia ligerica, Nereis sp.; Crustacés: Corophium volutator).

Le Gobie (Gobius microps) se reproduit au printemps et en été. La croissance des jeunes dans l'estuaire de la Loire est rapide de juin à octobre puis s'arrête en hiver. Pendant cette période, la plupart des Poissons adultes migre vers des eaux plus profondes. Les jeunes individus ont principalement une nourriture planctonique et utilisent surtout les Mysidacés (Neomysis integer) vivant près du fond. Au cours de la croissance, la nourriture devient plus benthique et comporte alors principalement des Annélides (Boccardia ligerica) et, dans la zone aval de l'estuaire surtout, des petits Crustacés (Crangon crangon, Corophium volutator) et des siphons de jeunes Mollusques (Mya arenaria).

Il est important de remarquer que, chez ces deux espèces, l'ingestion de faune endogée s'accompagne de l'ingestion de sédiments fins et de débris végétaux qui constituent un véritable "piège" pour les oligo-éléments étudiés. De ce fait, on a souvent avancé l'hypothèse que les sédiments pouvaient constituer un important vecteur de contamination pour la faune benthique.

Les super-carnivores sont représentés par trois espèces présentes occasionnellement dans l'estuaire: l'une d'eau douce, le Sandre (Stizostedion lucioperca), les deux autres marines, le Tacaud (Gadus luscus) et le Merlan (Merlangius merlangus). Les principales proies utilisées sont les Crevettes (Crangon crangon) et les Poissons de petite taille, Eperlan (Osmerus eperlanus) et Gobie (Gobius microps) (Marchand, 1978).

\section{MATERIEL ET METHODES}

Nous rappellerons brièvement le protocole expérimental qui a été décrit dans des publications antérieures (Boiteau \& Metayer, 1978; Amiard et al., 1980; Amiard-Triquet et al, 1980). Des prélèvements mensuels de zooplancton, d'ichthyofaune et de macrofaune benthique sont effectués dans l'estuaire interne de la Loire (France) sur les bancs de Bilho et de l'île Pipy respectivement distants de l'embouchure de 3,5 et $17 \mathrm{kms}$.

Les échantillons d'ichthyofaune ont été séparés en deux fractions qui sont ramenés rapidement au laboratoire en glacière. La première, fixée dans le formol, a servi à l'analyse faunistique des contenus digestifs; la seconde, congelée, à la mesure des concentrations en métaux.

Les échantillons formolés sont disséqués afin d'isoler le tube digestif. L'estomac et l'intestin sont séparés et leurs contenus extraits par pression et lavage. L'examen des 
fragments plus ou moins broyés permet la reconnaissance des espèces ingérées dont les abondances relatives sont déterminées par dénombrement des régions antérieures.

Les échantillons destinés à l'analyse des métaux sont décongelés. Les consommateurs (Poissons appartenant à cinq espèces) sont disséqués pour séparer le contenu digestif (en distinguant éventuellement contenu stomacal et contenu intestinal) de l'animal dont il ne fait pas partie intégrante. Pour les organismes-proies, le contenu digestif n'était pas séparé des tissus vivants, y compris pour les Poissons (Gobie, Eperlan) consommés par les super-prédateurs. Pour chaque espèce et pour chaque date de récolte, les échantillons étaient constitués par la réunion des prélèvements effectués sur trois individus au minimum.

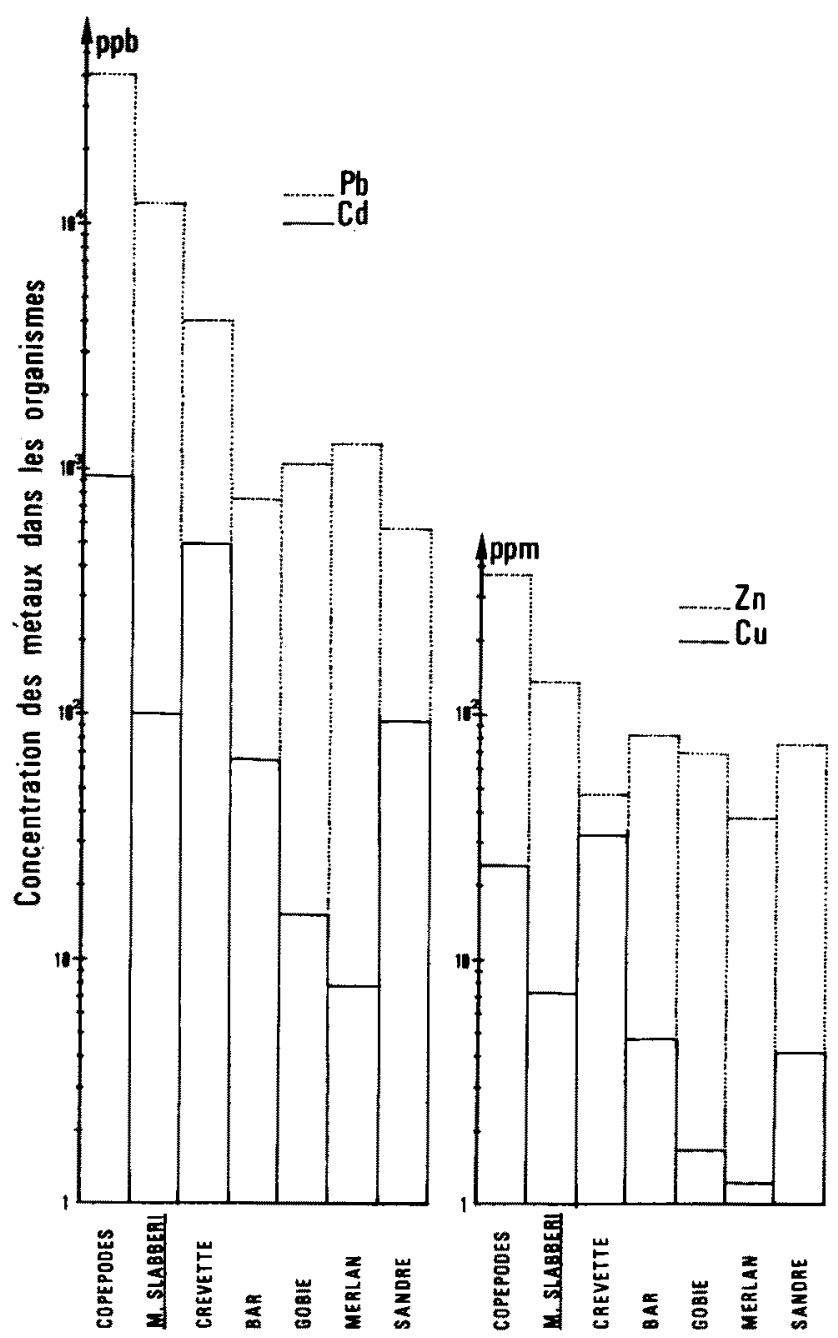

Fig. 1. Comparaison des concentrations métalliques moyennes détectées dans les organismes provenant du banc de l'île Pipy 
Tableau 1. Accumulation des métaux chez les Poissons omnivores de l'estua sont affectées de l'écart-type à la moyenn

\begin{tabular}{|c|c|c|c|c|c|c|}
\hline \multirow{2}{*}{$\begin{array}{l}\text { Espèces, lieux et } \\
\text { dates des prélè- } \\
\text { vements }\end{array}$} & \multirow{2}{*}{$\begin{array}{l}\text { Nombre } \\
\text { d'indi- } \\
\text { vidus }\end{array}$} & \multirow{2}{*}{$\begin{array}{c}\text { Poids } \\
\text { frais d'un } \\
\text { individu }(g)\end{array}$} & \multirow{2}{*}{$\frac{\text { Poids sec }}{\text { poids frais }}$} & \multirow{2}{*}{$\begin{array}{l}\text { Nature de la } \\
\text { nourriture }\end{array}$} & \multicolumn{2}{|c|}{ Teneur en $\mathrm{Cd}(\mu \mathrm{g} / \mathrm{kg} \mathrm{sec}) \mathrm{de}:$} \\
\hline & & & & & Nourriture & Consommateu \\
\hline \multicolumn{7}{|l|}{ Bar } \\
\hline \multicolumn{7}{|c|}{ (Dicentrarchus labrax) } \\
\hline B $29 / 08 / 78$ & 8 & 0,89 & 0,2337 & N. integer & $69,0 \pm$ & $115,7 \pm 3,9$ \\
\hline B $27 / 09 / 78$ & 9 & 1,46 & 0,2334 & C. crangon juv." & $140,1 \pm 18,2$ & $73,9 \pm 32,7$ \\
\hline B $25 / 10 / 78$ & 12 & 2,60 & 0,2361 & - & - & $13,8 \pm 2,3$ \\
\hline $\mathrm{P} 23 / 11 / 77$ & 7 & 4,29 & 0,2649 & Zooplancton $^{+}$ & $1146,1 \pm 18,3$ & $105,9 \pm 17,0$ \\
\hline P 27/07/78 & 3 & 0,26 & 0,2155 & Zooplancton $^{+}$ & $707,4 \pm 40,8$ & $48,3 \pm 4,0$ \\
\hline P $30 / 08 / 78$ & 3 & 1,50 & 0,2336 & - & - & $83,9 \pm 10,5$ \\
\hline P 26/09/78 & 12 & 2,42 & 0,2389 & - & - & $58,2 \pm 15,7$ \\
\hline P $27 / 10 / 78$ & 8 & 3,20 & 0,2490 & - & - & $27,0 \pm 9,1$ \\
\hline \multicolumn{7}{|l|}{ Gobie } \\
\hline B $4 / 10 / 77$ & 20 & 0,77 & 0,2288 & - & - & $41,7 \pm 4,0$ \\
\hline B $22 / 11 / 77$ & 20 & 0,55 & 0,2250 & C. crangon & $660,0 \pm 63,4$ & $24,4 \pm 3,9$ \\
\hline B $21 / 12 / 77$ & 20 & 0,68 & 0,2327 & B. ligerica & $4026,6 \pm 275,5$ & $102,1 \pm 9,6$ \\
\hline \multirow[t]{2}{*}{ B $26 / 07 / 78$} & 15 & 0,46 & 0,2368 & $N$. integer & $25,7 \pm$ & $<4,7$ \\
\hline & & & & C. crangon juv. & $94,1 \pm$ & \\
\hline \multirow[t]{2}{*}{ B $29 / 08 / 78$} & 20 & 0,62 & 0,2398 & $N$. integer & $69,0 \pm$ & $44,1 \pm 8,1$ \\
\hline & & & & C. crangon juv. & $102,6 \pm$ & \\
\hline B $25 / 10 / 78$ & 16 & 0,79 & 0,2363 & C. crangon juv." & $108,9 \pm 12,2$ & $<4,7$ \\
\hline P $10 / 10 / 77$ & 20 & 0,50 & 0,2223 & C. crangon" & $403,2 \pm 65,1$ & $23,4 \pm 2,3$ \\
\hline P $23 / 11 / 77$ & 20 & 0,57 & 0,2285 & Zooplancton *+ & $1146,1 \pm 18,3$ & $15,6 \pm 3,1$ \\
\hline P $22 / 12 / 77$ & 20 & 0,65 & 0,2239 & - & - & $20,8 \pm 3,2$ \\
\hline P $30 / 08 / 78$ & 20 & 0,41 & 0,2304 & - & - & - \\
\hline P 26/09/78 & 20 & 0,60 & 0,2275 & - & - & $17,9 \pm 5,6$ \\
\hline P $27 / 10 / 78$ & 20 & 0,92 & 0,2359 & - & - & $13,0 \pm 3,6$ \\
\hline
\end{tabular}

Les différents échantillons étaient ensuite soumis aux opération suivantes: (a) séchage à poids constant à l'étuve $\left(80^{\circ} \mathrm{C}\right)$; (b) réduction en poudre; (c) prélèvement de trois fractions aliquotes de $50 \mathrm{mg}$ environ; (d) minéralisation; (e) extraction à l'aide de dithizone en solution chloroformique; (f) dosage par spectrométrie d'absorption atomique de flamme $(\mathrm{Zn})$ ou sans flamme $(\mathrm{Cd}, \mathrm{Pb}, \mathrm{Cu})$.

\section{RESULTATS}

Les concentrations métalliques sont toujours exprimées par rapport au poids sec des tissus.

\section{Accumulation des métaux dans les organismes estuariens}

Les résultats détaillés sont présentés dans les Tableaux 1 et 2 . Pour chaque espèce, 
Loire et dans leur nourriture (les concentrations métalliques moyennes ranc de Bilho; $\mathrm{P}=$ banc de l'île Pipy

\begin{tabular}{|c|c|c|c|c|c|c|}
\hline \multicolumn{3}{|c|}{ Teneur en $\mathrm{Pb}(\mu \mathrm{g} / \mathrm{kg} \mathrm{sec})$} & \multicolumn{2}{|c|}{ Teneur en $\mathrm{Cu}(\mu \mathrm{g} / \mathrm{kg} \mathrm{sec})$} & \multicolumn{2}{|c|}{ Teneur en $\mathrm{Zn}(\mathrm{mg} / \mathrm{kg} \mathrm{sec})$} \\
\hline Nourrit & ure & Consommateur & Nourriture & Consommateur & Nourriture & Consommateur \\
\hline$: 1,7 \pm$ & 239,4 & $584,4 \pm$ & $20492,4 \pm 872,0$ & $3306,2 \pm 69,3$ & $47,8 \pm 1,0$ & $81,5 \pm 1,3$ \\
\hline $13,2 \pm$ & 114,5 & $605,8 \pm 85,5$ & $38638,1 \pm 4355,4$ & $3502,1 \pm 460,2$ & $46,1 \pm 1,5$ & $79,0 \pm 5,0$ \\
\hline - & & $1221,7 \pm 499,5$ & - & $2675,7 \pm 216,9$ & - & $81,6 \pm 2,2$ \\
\hline $18,4 \pm$ & 170,6 & $415,5 \pm 52,6$ & $31290,9 \pm 2931,1$ & $4167,4 \pm 332,6$ & $288,3 \pm 16,7$ & $68,9 \pm 9,1$ \\
\hline $.1,4 \pm 1$ & 4123,0 & $656,3 \pm 89,6$ & $17753,0 \pm 575,9$ & $4853,2 \pm 97,1$ & $447,9 \pm 19,8$ & $101,3 \pm 4,0$ \\
\hline - & & $381,1 \pm 69,2$ & - & $4297,8 \pm 433,7$ & - & $87,7 \pm 5,3$ \\
\hline- & & $1257,5 \pm 189,4$ & - & $2663,8 \pm 496,1$ & - & $78,4 \pm 3,8$ \\
\hline- & & $1057,6 \pm 107,4$ & - & $2303,4 \pm 211,8$ & - & $76,9 \pm 6,1$ \\
\hline- & & $1095,0 \pm 181,3$ & - & $1206,9 \pm 100,3$ & - & $62,4 \pm 2,8$ \\
\hline $15,3 \pm$ & 837,7 & $977,2 \pm 138,1$ & $36415,3 \pm 2569,0$ & $1024,7 \pm 216,3$ & $44,6 \pm 2,6$ & $68,5 \pm 7,0$ \\
\hline $14,6 \pm$ & 726,8 & $1542,7 \pm 117,5$ & $12897,7 \pm 831,2$ & $2031,3 \pm 142,7$ & $245,1 \pm 8,2$ & $82,6 \pm 6,0$ \\
\hline $16,0 \pm$ & 575,6 & $1415,8 \pm 191,6$ & $35256,6 \pm 1563,3$ & $2332,2 \pm 454,1$ & $64,1 \pm 4,4$ & $68,2 \pm 2,8$ \\
\hline $3,5 \pm$ & 248,8 & & $8415,9 \pm 1159,7$ & & $36,5 \pm 2,0$ & \\
\hline$\$ 1,7 \pm$ & 239,4 & $359,7 \pm 99,8$ & $20492,4 \pm 872,0$ & $1203,1 \pm 146,6$ & $47,8 \pm 1,0$ & $58,0 \pm 3,9$ \\
\hline $19,8 \pm$ & 100,9 & & $22425,1 \pm 1412,5$ & & $39,0 \pm 0,2$ & \\
\hline $13,9 \pm$ & 156,7 & $2055,1 \pm 106,1$ & $37123,7 \pm 1042,2$ & $1024,6 \pm 101,4$ & $47,3 \pm 1,7$ & $67,6 \pm 7,7$ \\
\hline $15,1 \pm$ & 359,3 & $670,6 \pm 33,5$ & $29902,9 \pm 1152,3$ & $1510,9 \pm 134,5$ & $41,7 \pm 2,7$ & $79,1 \pm 4,2$ \\
\hline $38,4 \pm$ & 170,6 & $725,7 \pm 38,7$ & $31290,9 \pm 2931,1$ & $1704,1 \pm 139,8$ & $288,3 \pm 16,7$ & $81,6 \pm 4,3$ \\
\hline- & & $1096,5 \pm 22,9$ & - & $2228,4 \pm 127,1$ & - & $67,3 \pm 2,2$ \\
\hline - & & $903,2 \pm 72,8$ & - & $1158,9 \pm 61,9$ & - & $55,8 \pm 3,0$ \\
\hline - & & $1321,4 \pm 36,9$ & - & $1792,0 \pm 97,0$ & - & $73,2 \pm 13,0$ \\
\hline- & & $1523,0 \pm 275,7$ & - & $1668,9 \pm 162,2$ & - & $55,9 \pm 3,0$ \\
\hline
\end{tabular}

nous avons de plus calculé les moyennes des concentrations métalliques détectées au cours de prélèvements successifs (Fig. 1 et 2).

Les concentrations les plus élevées de $\mathrm{Cd}$ et de $\mathrm{Pb}$ se rencontrent chez les Annélides suivis par les Crustacés, les niveaux les plus faibles étant observés chez les Poissons. Nous notons une seule inversion pour $\mathrm{Cd}$ entre le Bar et les Mysidacés (Neomysis integer) prélevés sur le banc de Bilho.

Le cuivre s'accumule surtout chez les Crustacés: sur le banc de Bilho, Crangon crangon et Neomysis integer présentent des concentrations sensiblement doubles de celles de Boccardia ligerica et environ dix fois plus élevées que celles des Poissons. Les trois échantillons de Crustacés provenant du banc de l'île Pipy présentent des teneurs en cuivre nettement plus élevées que les quatre espèces de Poissons.

Les teneurs en $\mathrm{Zn}$ sont du même ordre de grandeur chez les Crustacés (à l'exception des Copépodes) et les Poissons. Elles sont nettement plus fortes chez les Annélides et les Copépodes. 
Tableau 2. Accumulation des métaux chez les Poissons super-carnivores moyennes sont affectées de l'écart-type à

\begin{tabular}{|c|c|c|c|c|c|c|}
\hline \multirow{2}{*}{$\begin{array}{l}\text { Espèces, lieux et } \\
\text { dates des prélè- } \\
\text { vements }\end{array}$} & \multirow{2}{*}{$\begin{array}{l}\text { Nombre } \\
\text { d'indi- } \\
\text { vidus }\end{array}$} & \multirow{2}{*}{$\begin{array}{c}\text { Poids } \\
\text { frais d'un } \\
\text { individu }(g)\end{array}$} & \multirow{2}{*}{$\frac{\text { Poids sec }}{\text { poids frais }}$} & \multirow{2}{*}{$\begin{array}{c}\text { Nature de la } \\
\text { nourriture }\end{array}$} & \multicolumn{2}{|c|}{ Teneur en $\mathrm{Cd}(\mu \mathrm{g} / \mathrm{kg} \mathrm{sec}) \mathrm{de}$} \\
\hline & & & & & Nourriture & Consommateur \\
\hline \multicolumn{7}{|c|}{$\begin{array}{l}\text { Merlañ } \\
\text { (Merlangius merlangus) }\end{array}$} \\
\hline B $26 / 07 / 78$ & 6 & 1,72 & 0,2087 & $\begin{array}{l}C . \text { crangon } \\
N \text {. integer }\end{array}$ & $\begin{array}{l}94,1 \pm 8,0 \\
25,7 \pm 4,2\end{array}$ & $4,9 \pm 3,2$ \\
\hline B $29 / 08 / 78$ & 12 & 3,53 & 0,1910 & $N$. integer & $69,0 \pm 5,7$ & $36,5 \pm 3,2$ \\
\hline P $27 / 10 / 78$ & 10 & 7,13 & 0,1888 & $\begin{array}{l}\text { C. crangon } \\
\text { M. slabberi } \\
\text { G. microps } \\
\text { O. eperlanus }\end{array}$ & $\begin{array}{c}112,5 \pm 14,7 \\
97,8 \pm 25,1 \\
16,1 \\
5,7\end{array}$ & $7,8 \pm 6,2$ \\
\hline \multicolumn{7}{|l|}{$\begin{array}{l}\text { Tacaud } \\
\text { (Gadus Iuscus) }\end{array}$} \\
\hline B $26 / 06 / 78$ & 6 & 3,29 & 0,2034 & C. crangon & $183,3 \pm 30,2$ & $14,8 \pm 1,1$ \\
\hline \multicolumn{7}{|c|}{$\begin{array}{l}\text { Sandre } \\
\text { (Stizostedion lucioperca) }\end{array}$} \\
\hline $\mathrm{P} 11 / 08 / 77$ & 7 & 2,18 & 0,2105 & $\begin{array}{l}\text { G.microps } \\
\text { O. eperlanus } \\
\text { C. crangon }\end{array}$ & $\begin{array}{c}74,2 \pm 2,1 \\
24,19 \\
966,1 \pm 96,8\end{array}$ & $93,4 \pm 10,9$ \\
\hline
\end{tabular}

A quelques exceptions près entre $\mathrm{Cu}$ et $\mathrm{Pb}$, la hiérarchie des métaux en fonction de leur accumulation biologique est la suivante:

$$
\mathrm{Cd}<\mathrm{Pb} \leq \mathrm{Cu}<\mathrm{Zn}
$$

\section{Transfert des métaux dans les chaînes alimentaires}

Afin de quantifier ce transfert, nous calculerons un facteur de transfert $f$. $t$ défini (Amiard \& Amiard-Triquet, 1977), en première approximation, comme le rapport:

$$
\text { f. } \mathrm{t} .=\frac{\text { concentration métallique dans le consommateur }}{\text { concentration métallique dans la proie }}
$$

L'analyse des contenus digestifs indique que certaines espèces prédatrices utilisent en proportions variables $\left(p_{1}, p_{2}, \ldots p_{x}\right.$ en pourcentage) plusieurs espèces-proies pouvant présenter des niveaux de concentration différents: $n_{1}, n_{2}, \ldots, n_{x}$ (Tabl. 1 et 2). Dans ce cas, nous avons calculé un facteur de transfert pondéré:

$$
\text { f. } \mathrm{t} .=\frac{\text { concentration dans le consommateur }}{\frac{\left(\mathrm{n}_{1} \times \mathrm{p}_{1}\right)+\left(\mathrm{n}_{2} \times \mathrm{p}_{2}\right)+\ldots+\left(\mathrm{n}_{\mathrm{x}} \times \mathrm{p}_{\mathrm{x}}\right)}{\mathrm{p}_{1}+\mathrm{p}_{2}+\ldots+\mathrm{p}_{\mathrm{x}}}}
$$

Les facteurs de transfert moyens calculés pour chaque espèce et pour chaque métal sont présentés dans le Tableau 3. 
stuaire de la Loire et dans leur nourriture (les concentrations métalliques

wrenne). $\mathrm{B}=$ banc de Bilho; $\mathrm{P}=$ banc de l'île Pipy

\begin{tabular}{|c|c|c|c|c|c|}
\hline \multicolumn{2}{|c|}{ Teneur en $\mathrm{Pb}(\mu \mathrm{g} / \mathrm{kg} \mathrm{sec})$ de: } & \multicolumn{2}{|c|}{ Teneur en $\mathrm{Cu}(\mu \mathrm{g} / \mathrm{kg} \mathrm{sec})$ de: } & \multicolumn{2}{|c|}{ Teneur en $\mathrm{Zn}(\mathrm{mg} / \mathrm{kg}$ sec) de: } \\
\hline Nourriture & Consommateur & Nourriture & Consommateur & Nourriture & Consommateur \\
\hline $2873,5 \pm 248,8$ & $1741,0 \pm 189,8$ & $8415,9 \pm 1159,7$ & $2944,8 \pm 678,5$ & $36,5 \pm 2,0$ & $32,9 \pm 1,4$ \\
\hline $4046,0 \pm 575,6$ & & $35256,6 \pm 1563,3$ & & $64,1 \pm 4,4$ & \\
\hline $4651,7 \pm 239,4$ & $693,4 \pm 44,5$ & $20492,4 \pm 872,0$ & $1261,1 \pm 137,3$ & $47,8 \pm 1,0$ & $50,1 \pm 2,7$ \\
\hline $5507,2 \pm 120,3$ & $1248,3 \pm 79,3$ & $34000,3 \pm 2472,7$ & $1227,8 \pm 141,1$ & $40,4 \pm 1,7$ & $38,2 \pm 5,5$ \\
\hline $\begin{array}{c}12053,6 \pm 489,7 \\
1684,8\end{array}$ & & $\begin{array}{c}7257,2 \pm 1941,3 \\
1802,1\end{array}$ & & $\begin{array}{c}134,0 \pm 14,2 \\
56,3\end{array}$ & \\
\hline $\begin{array}{c}4255,6 \pm 538,2 \\
-\end{array}$ & $\begin{array}{r}1796,4 \pm 552,4 \\
275,1 \pm 38,0\end{array}$ & $\begin{aligned} 5970,2 & \pm 645,4 \\
& -\end{aligned}$ & $\begin{array}{l}2881,6 \pm 477,5 \\
1381,2 \pm 201,9\end{array}$ & $\begin{aligned} 36,4 & \pm 1,9 \\
- & \end{aligned}$ & $\begin{array}{l}29,5 \pm 4,1 \\
44,4 \pm 3,7\end{array}$ \\
\hline $\begin{array}{c}1449,5 \pm 104,5 \\
1884,9 \\
3214,8 \pm 167,6\end{array}$ & $573,1 \pm 32,4$ & $\begin{array}{c}2138,5 \pm 313,3 \\
3465,4 \\
33471,5 \pm 4216,3\end{array}$ & $3093,4 \pm 66,2$ & $\begin{array}{c}72,6 \pm 5,2 \\
74,62 \\
51,5 \pm 3,0\end{array}$ & $75,7 \pm 2,6$ \\
\hline
\end{tabular}

Pour $\mathrm{Cd}, \mathrm{Pb}$ et $\mathrm{Cu}$, ils sont exceptionnellement supérieurs à 1 et ils sont les plus souvent inférieurs à 0,5 . Sur quinze $f$. $t$. utilisés pour le calcul des valeurs moyennes, on relève deux $\mathrm{f}$. $\mathrm{t}$. supérieurs à 1 pour $\mathrm{Cd}$ (Bar et Sandre), un f. t. supérieur à 1 pour $\mathrm{Cu}$ (Sandre) et aucune exception pour $\mathrm{Pb}$.

Pour $\mathrm{Zn}$, les facteurs de transfert moyens avoisinent (Bar, Merlan, Tacaud) ou dépassent légèrement 1 (Gobie, Sandre). Sur les quinze f. t. utilisés au total pour calculer les moyennes, neuf sont supérieurs à 1 .

Evolution de la concentration métallique dans les aliments au cours de la digestion

Nous avions proposé antérieurement (Amiard et al., 1980; Amiard-Triquet et al., 1980) de quantifier cette évolution à l'aide de deux rapports:

concentration métallique dans la nourriture

concentration métallique dans le contenu stomacal (ou digestif)

et

$$
\frac{\text { concentration métallique dans le contenu stomacal }}{\text { concentration métallique dans le contenu intestinal }}
$$

Dans le cas de Poissons mangeurs de sédiment comme le Bar et le Gobie, le premier de ces rapports ne présente pas d'intérêt dans la mesure où nous connaissons la 


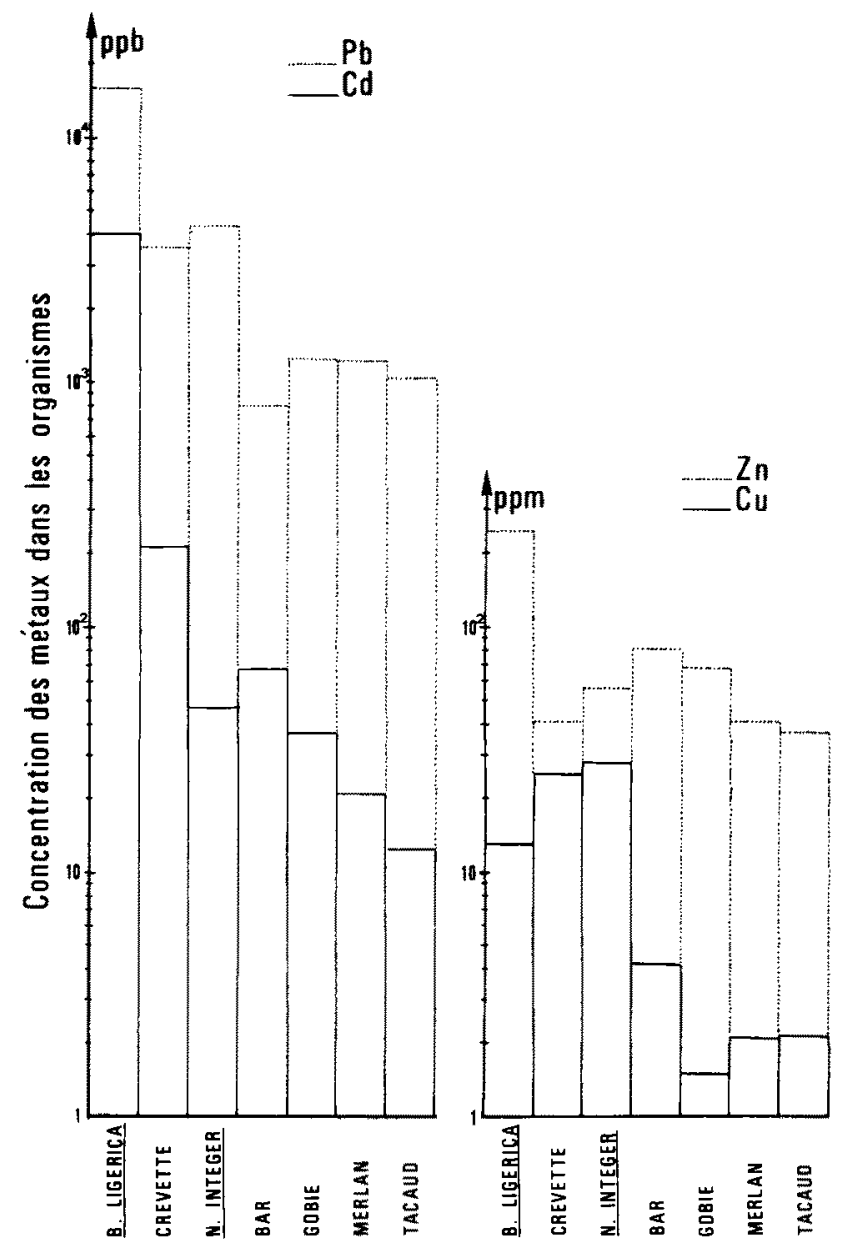

Fig. 2. Comparaison des concentrations métalliques moyennes détectées dans les organismes provenant du banc de Bilho

concentration des métaux dans les proies mais non dans le sédiment ingéré. Pour le Merlan, il varie de 0,86 à 2,60 selon les prélèvements pour $\mathrm{Cd}_{i}$ de 1,04 à 1,26 pour $\mathrm{Pb}$; de 1,20 à 2,65 pour $\mathrm{Cu}$ et de 0,80 à 0,88 pour $\mathrm{Zn}$.

La valeur du second rapport a pu être établie pour le Bar et le Merlan (Tabl. 4). Nous constatons qu'à une exception près (pour $\mathrm{Cu}$ ) ce rapport est inférieur à l'unité et même le plus souvent inférieur à 0,6 .

\section{DISCUSSION ET CONCLUSIONS}

Nos résultats corroborent l'observation fréquemment renouvelée d'une diminution des concentrations métalliques lorsque l'on considère les espèces les plus évoluées du point de vue zoologique (Poissons < Crustacés < Annélides). Il faut remarquer cepen- 
Tableau 3. Transfert des métaux dans les chaînes alimentaires de l'estuaire de la Loire. $\bar{x}=$ moyenne établie sur l'ensemble des prélèvements; $\left(s_{m}\right)=$ écart-type à la moyenne

\begin{tabular}{|c|c|c|c|c|}
\hline \multirow[b]{2}{*}{ Espèces } & \multicolumn{4}{|c|}{ Facteurs de transfer de } \\
\hline & $\mathrm{Cd}$ & $\mathrm{Pb}$ & $\mathrm{Cu}$ & $\mathrm{Zn}$ \\
\hline \multicolumn{5}{|l|}{ Bar } \\
\hline $\bar{x}\left(s_{m}\right)$ & $0,59 \quad(0,38)$ & $0,09 \quad(0,05)$ & $0,16 \quad(0,04)$ & $0,97 \quad(0,43)$ \\
\hline valeurs-limites & $0,07-1,68$ & $0,01-0,22$ & $0,09-0,27$ & $0,23-1,71$ \\
\hline \multicolumn{5}{|l|}{ Gobie } \\
\hline$\overline{\mathrm{x}}\left(\mathrm{s}_{\mathrm{m}}\right)$ & $0,15 \quad(0,10)$ & $0,21 \quad(0,06)$ & $0,07 \quad(0,02)$ & $1,14 \quad(0,23)$ \\
\hline valeurs-limites & $0,01-0,63$ & $0,04-0,42$ & $0,03-0,16$ & $0,28-1,90$ \\
\hline \multicolumn{5}{|l|}{ Merlan } \\
\hline$\overline{\mathrm{x}}\left(\mathrm{s}_{\mathrm{m}}\right)$ & $0,37 \quad(0,11)$ & $0,30 \quad(0,10)$ & $0,16 \quad(0,06)$ & $0,74 \quad(0,16)$ \\
\hline valeurs-limites & $0,15-0,53$ & $0,15-0,48$ & $0,06-0,26$ & $0,55-1,05$ \\
\hline Tacaud & 0,08 & 0,42 & 0,48 & 0,81 \\
\hline Sandre & 1,63 & 0,37 & 1,32 & 1,05 \\
\hline
\end{tabular}

dant l'accumulation préférentielle du cuivre chez les Crustacés que l'on peut rapprocher de la présence de ce métal dans l'hémocyanine, pigment respiratoire des Crustacés. Si les teneurs en zinc les plus élevées sont détectées chez les Annélides et les Copépodes, elles sont du même ordre de grandeur chez les Crevettes, les Mysidacées (Crustacés Malacostracés) et les Poissons (Bernhard \& Zattera, 1975; Amiard et al, 1980; AmiardTriquet et al., 1980). L'élimination du contenu digestif des Poissons, qui présente généralement des concentrations métalliques beaucoup plus élevées que les tissus, augmente artificiellement les différences relevées avec les autres groupes zoologiques. De même, les échantillons de zooplancton peuvent être "contaminés" par des particules inorganiques (argiles en particulier) riches en métaux (Flegal \& Martin, 1977; AmiardTriquet et al., à paraître).

Nous avons comparé nos données avec celles fournies par la littérature pour les Tableau 4. Evolution des teneurs en métaux du contenu digestif au cours de la digestion. $\mathrm{B}=$ banc de Bilho; $\mathrm{P}=$ banc de l'île Pipy

\begin{tabular}{|llccc|}
\hline $\begin{array}{l}\text { Espèce, date et lieu } \\
\text { de prélèvement }\end{array}$ & \multicolumn{5}{c|}{ Rapport contenu stomacal/contenu intestinal } \\
& $\mathrm{Cd}$ & $\mathrm{Pb}$ & $\mathrm{Cu}$ & $\mathrm{Zn}$ \\
\hline $\mathrm{Bar}$ & & & & \\
$\mathrm{B} 29 / 08 / 78$ & 0,54 & 0,48 & 0,68 & 0,39 \\
$\mathrm{~B} 27 / 09 / 78$ & 0,34 & 0,56 & 0,72 & 0,42 \\
$\mathrm{~B} 25 / 10 / 78$ & 0,24 & 0,39 & 0,53 & 0,32 \\
$\mathrm{P} 26 / 09 / 78$ & 0,51 & 0,62 & 1,08 & 0,50 \\
$\mathrm{P} 27 / 10 / 78$ & 0,57 & 0,75 & 0,90 & 0,67 \\
Merlan & & & & \\
$\mathrm{P} 27 / 10 / 78$ & 0,05 & 0,15 & 0,17 & 0,36 \\
\hline
\end{tabular}


mêmes espèces ou des espèces voisines prélevées dans des zones polluées ou non. $\mathrm{A}$ l'exception de la teneur en Cd relativement élevée de $B$. ligerica par rapport à Nereis diversicolor provenant de différents estuaires britanniques (Bryan \& Hummerstone, 1973, 1977; Wharfe \& van den Broek, 1977), les concentrations déterminées dans l'estuaire de la Loire constituent fréquemment des valeurs intermédiaires entre les valeurs-limites relevées dans la littérature. Dans de nombreux cas ( $\mathrm{Cu}$ chez $B$. ligerica, $\mathrm{Cd}$ chez les Copépodes, $\mathrm{Cd}$ et $\mathrm{Pb}$ chez $\mathrm{C}$. crangon, $\mathrm{Cd}, \mathrm{Pb}$ et $\mathrm{Cu}$ chez les Merlans et $\mathrm{Cd}$, $\mathrm{Pb}, \mathrm{Cu}$ et $\mathrm{Zn}$ chez les Gobies), les organismes de l'estuaire de la Loire présentent des valeurs plus faibles que ceux provenant dautres aires géographiques (Bryan, 1968; Bryan \& Hummerstone, 1971, 1973, 1977; Halcrow et al., 1973; Peden et al., 1973; Steele et al., 1973; Hardisty et al., 1974a et b, Bohn \& Mc Elroy, 1976; Wright, 1976; Badsha \& Sainsbury, 1977; Greig et al., 1977; Wharfe \& van den Broek, 1977).

Le niveau d'évolution des espèces (considéré en fonction de la classification zoologique) varie fréquemment comme le niveau trophique. Les Annélides et les Crustacés, qui constituent l'essentiel des proies, présentant généralement des concentrations en $\mathrm{Cd}, \mathrm{Pb}$ et $\mathrm{Cu}$ plus élevées que celles mesurées chez les Poissons qui les consomment, il en résulte des facteurs de transfert presque toujours inférieurs à l'unité. Pour les organismes (Bar, Gobie) qui ingèrent du sédiment avec leur proie, nous n'avons pas fait intervenir cette "nourriture" de nature très particulière dans le calcul du facteur de transfert. En effet, bien que la disponibilité biologique des polluants massivement liés à la phase sédimentaire soit un des problèmes majeurs de l'écologie appliquée à la protection de l'Homme et de son environnement, les mécanismes et l'importance quantitative de ce phénomène sont très mal connus. A court terme, les quantités mises en jeu dans les échanges sédiment $\rightarrow$ organismes semblent très faibles par rapport à celles concernant les échanges eau ou nourriture $\rightarrow$ organismes. A long terme par contre, on évalue mal les conséquences d'un transfert direct (sédiment $\rightarrow$ organisme) ou indirect (sédiment $\rightarrow$ eau $\rightarrow$ organisme) (Amiard-Triquet, 1975; Jenne \& Luoma, 1977; Luoma \& Jenne, 1977).

Les données de la littérature résultant soit de mesures in situ de la concentration des métaux en fonction du niveau trophique des organismes, soit d'expériences de contamination des organismes par voie alimentaire, confirment que s'il y a une bioaccumulation* non négligeable de $\mathrm{Cd}, \mathrm{Pb}$ et $\mathrm{Cu}$ chez les organismes aquatiques, il n'y a vraisemblablement pas biomagnification* " dans les réseaux alimentaires (Bohn \& Mc Elroy, 1976; Aubert et al., 1972, 1974, 1975, 1976; Drifmeyer \& Odum, 1975; Rehwoldt et al., 1978; Hodson et al, 1978; Steele et al., 1973).

Pour le zinc, nos résultats ne permettent pas de conclusions aussí nettes et il en est de même des observations in situ ou au laboratoire des autres auteurs pour le milieu marin (in: Amiard-Triquet et al., 1980). Les concentrations relativement élevées détectées chez les consommateurs peuvent-elles résulter d'un stockage cumulatif du zinc à long terme? Dans ce cas, on devrait observer une accumulation plus forte chez les animaux âgés que chez les jeunes. Dans l'estuaire de la Loire, en période printanière,

\footnotetext{
- Bioaccumulation: accumulation dans les organismes résultant soit d'une contamination directe par l'eau (adsorption ou absorption), soit d'une contamination indirecte par voie alimentaire.

* Biomagnification: bioaccumulation caractérisée par une augmentation de la concentration dans les prédateurs par rapport à leurs proies.
} 
nous avons observé une corrélation faiblement négative $(r=-0,53)$ entre l'âge des Flets ( 6 mois à 5 ans) et leur teneur en zinc (déterminée sur l'animal in toto dont le contenu digestif a été éliminé) (Amiard et al., non publié). D'une manière générale, les données de la littérature sont très hétérogènes sur ce point (in: Pentreath, 1977). On a même observé pour une même espèce, prélevée dans un même lieu, des résultats contradictoires d'une saison à l'autre (Hardisty et al., 1974a).

Dans l'estuaire de la Columbia, Renfro (1972) a observé que la vitesse d'élimination du zinc était d'autant plus faible que l'on s'adressait à un organisme de niveau trophique plus élevé. De plus, expérimentalement, Baptist et al. (1970) et Baudin (1977) ont montré que la vitesse d'élimination du zinc était plus faible après contamination par la nourriture qu'après contamination par l'eau. Ces deux phénomènes en se conjuguant pourraient expliquer une accumulation préférentielle du zinc dans les organismes des niveaux trophiques les plus élevés.

Il ne faut pas négliger le rôle de l'eau comme vecteur de l'accumulation du zinc. En effet, si les principaux organes de stockage de ce métal chez les Poissons sont les organes génitaux, le foie, les reins et les os, la peau présente également des teneurs élevées (Hiyama \& Shimizu, 1964; Halcrow et al., 1973; Merlini et al., 1973; Pentreath, 1973; Ting, 1973; Ishikawa \& Ohno, 1974; Wright, 1976; Wharfe \& van den Broek, 1977; Amiard et al., non publié) peut-être liées à la présence du mucus qui possède une importante capacité de rétention du zinc (Pentreath, 1973; Coombs et al., 1972 in Pentreath, 1977).

\section{LITERATURE CITEE}

Amiard, J.-C. \& Amiard-Triquet, C., 1977. Health and ecological aspects of cobalt 60 transfer in a seawater food chain typical of an intertidal mud-flat. - Int. J. environ. Stud. 10, 113-118.

Amiard, J.-C., Amiard-Triquet, C., Métayer, C. \& Marchand, J., 1980. Etude du transfert de Cd, Pb, $\mathrm{Cu}$ et $\mathrm{Zn}$ dans les chaînes trophiques néritiques et estuariennes. 1. Etat dans l'estuaire interne de la Loire (France) au cours de l'été 1978. - Wat. Res. 14, 665-673.

Amiard-Triquet, C., 1975. Etude du transfert des radionucléides entre le milieu sédimentaire marin et les invertébrés qui y vivent. Thèse d'Etat, Université de Nantes, $99 \mathrm{pp}$.

Amiard-Triquet, C. Métayer, C. \& Amiard, J.-C., 1980. Etude du transfert de Cd, $\mathrm{Pb}, \mathrm{Cu}$ et $\mathrm{Zn}$ dans les chaînes trophiques néritiques et estuariennes. 2. Accumulation biologique chez les Poissons planctonophages. - Wat. Res. 14, 1327-1332.

Aubert, M., Bittel, R., Laumond, F., Roméo, M., Donnier, B. \& Barelli, M., 1972. Utilisation d'une chaine trophodynamique de type pélagique pour l'étude des transferts des pollutions métalliques. - Revue int. Océanogr. méd. 28, 27-52.

Aubert, M., Bittel, R., Laumond, F., Roméo, M., Donnier, B. \& Barelli, M., 1974. Utilisation d'une chaîne trophodynamique de type néritique á Mollusque pour l'étude des transferts des polluants métalliques. - Revue int. Océanogr. méd. 33, 7-29.

Aubert, M., Bittel, R., Laumond, F., Barelli, M., Gauthier, M., Donnier, B. \& Romeo, M., 1975. Utilisation d'une chaîne trophodynamique de type benthique pour l'étude des transferts des polluants métalliques. Revue int. Océanogr. méd. 39-40, 117-151,

Aubert, M., Laumond, F., Flatau, G., Barelli, M., Roméo, M. \& Puel, D., 1976. Utilisation d'une chaîne trophodynamique marine de type néritique à Crustacés pour l'étude du transfert et de la accumulation de divers polluants métalliques. - Revue int. Océanogr. méd. 43, 47-63.

Badsha, K. S. \& Sainsbury, M., 1977. Uptake of zinc, lead and cadmium by young whiting in the Severn estuary. - Mar. Pollut. Bull. 8, 164-166.

Baptist, J. P., Hoss, D. E. \& Lewis, C. W., 1970. Retention of ${ }^{51} \mathrm{Cr},{ }^{59} \mathrm{Fe},{ }^{60} \mathrm{Co},{ }^{65} \mathrm{Zn},{ }^{85} \mathrm{Sr},{ }^{95} \mathrm{Nb},{ }^{114 m} \mathrm{In}$ and ${ }^{131}$ I by the Atlantic croaker (Micropogon undulatus). - Hith Phys. 18, 141-148.

Baudin, J.-P., 1977. Etude expérimentale des modalités du transfert du zinc-65 entre les principaux 
constituants d'un écosystème saumâtre (l'étang de Citis). Thèse d'Etat, Université d'AixMarseille II, $265 \mathrm{pp}$.

Bernhard, M. \& Zattera, A., 1975. Major pollutants in the marine environment. In: Marine pollution and marine waste disposal. Ed. by E. A. Pearson \& E. de Fraja Frangipane. Pergamon Press, New-York, 195-300.

Bohn, A. \& Mc Elroy, R. O., 1976. Trace metals (As, Cd, Cu, Fe, and $\mathrm{Zn}$ ) in Arctic cod, Boreogadus saida, and selected zooplankton from Strathcona Sound, northern Baffin Island. - J. Fish. Res. Bd Can. 33, 2836-2840.

Boiteau, H. L. \& Métayer, C., 1978. Microdosage du plomb, du cadmium, du zinc et de l'étain dans les milieux biologiques par spectrométrie d'absorption atomique après minéralisation et extraction. - Analusis 6, 350-358.

Bryan, G. W., 1968. Concentrations of zinc and copper in the tissues of decapod crustaceans. - J. mar. biol. Ass. U. K. 48, 303-321.

Bryan, G. W. \& Hummerstone, L. G., 1971. Adaptation of the polychaete Nereis diversicolor to estuarine sediments containing high concentrations of heavy metals. I. General observations and adaptation to copper. - J. mar. biol. Ass. U. K. 51, 845-863.

Bryan, G. W. \& Hummerstone, L. G., 1973. Adaptation of the polychaete Nereis diversicolor to estuarine sediments containing high concentrations of zinc and cadmium. - J. mar. biol. Ass. U. K. 53, 839-857.

Bryan, G. W. \& Hummerstone, L. G., 1977. Indicators of heavy-metal contamination in the Looe estuary (Cornwall) with particular regard to silver and lead. - J. mar. biol. Ass. U. K. 57, 75-92.

Drifmeyer, J. E. \& Odum, W. E, 1975. Lead, zinc and manganese in dredgespoil pond ecosystems in Virginia. - Environ. Conserv. 1, 1-7.

Flegal, A. R. \& Martin, J. H., 1977. Contamination of biological samples by ingested sediment. Mar. Pollut. Bull. 8, 90-92.

Greig, R. A., Adams, A. \& Wenzloff, D. R., 1977. Trace metal content of plankton and zooplankton collected from the New-York Bight and Long Island Sound. - Bull. environ. Contam. Toxicol. 18, 3-8.

Halcrow, W., Mackay, D. W. \& Thornton, I., 1973. The distribution of trace metals and fauna in the Firth of Clyde in relation to the disposal of sewage sludge. - J. mar. biol. Ass. U. K. 53, 721-739.

Hardisty, M. W., Huggins, R. J., Kartar, S. \& Sainsbury, M., 1974a. Ecological implications of heavy metal in fish from the Severn estuary. - Mar. Pollut. Bull. 5, 12-15.

Hardisty, M. W., Kartar, S. \& Sainsbury, M., 1974b. Dietary habits and heavy metal concentrations in fish from the Severn estuary and Bristol Channel. - Mar. Pollut. Bull. 5, 61-63.

Hiyama, Y. \& Shimizu, M., 1964. On the concentration-factors of radioactive Cs, Sr, Cd, Zn and Ce in marine organisms. - Rec. oceanogr. Wks Jap. 7, 43-77.

Hodson, P. V., Blunt, B. R. \& Spry, D. J., 1978. Chronic toxicity of waterborne and dietary lead to rainbow trout (Salmo gairdneri) in lake Ontario water. - Wat. Res. 12, 869-878.

Ichikawa, R. \& Ohno, S., 1974. Levels of cobalt, cesium and zinc in some marine organisms in Japan. - Bull. Jap. Soc. scient. Fish. 40, 501-508.

Jenne, E. A. \& Luoma, S. N., 1977. Forms of trace elements in soils, sediments and associated water: an overview of their determination and biological availability. In: Biological implications and metals in the environment. Ed. by R. Wildung \& H. Drucker. ERDA Publ, Washington, 110-143.

Luoma, S. N. \& Jenne, E. A., 1977. The avaibility of sediment-bound cobalt, silver and zinc to a deposit-feeding clam. In: Biological implications of metals in the environment. Ed by R. Wildung \& H. Drucker. ERDA Publ., Washington, 213-230.

Marchand, J., 1978. Etude de l'ichthyofaune de l'estuaire de la Loire. In: Etude écologique de la Basse-Loire de Nantes à Saint-Nazaire (Invertébrés - Vertébrés). Université de Nantes, Laboratoire de Biologie Marine, 79-217.

Merlini, M., Argentesi, F., Berg, A., Brazzelli, A., Oregioni, B. \& Pozzi, G., 1973. The biological pathway of zinc $\left({ }^{65} \mathrm{Zn}\right)$ in freshwater fish and its alteration by heavy metals. In: Radionuclides in ecosystems. Ed, by D. J. Nelson. U. S. A. E. C., Oak Ridge, Tenn., 285-306.

Peden, J. D., Crothers, J. H., Waterfall, C. E. \& Beasley, J., 1973. Heavy metals in Somerset marine organisms. - Mar. Pollut. Bull. 4, 7-9.

Pentreath, R. J., 1973. The accumulation and retention of ${ }^{65} \mathrm{Zn}$ and ${ }^{54} \mathrm{Mn}$ by the plaice, Pleuronectes platessa L.-J. exp. mar. biol. Ecol. 12, 1-18. 
Pentreath, R. J., 1977. Radionuclides in marine fish. - Oceanogr. mar. Biol. 15, 365-460.

Rehwoldt, R. E., Mastrianni, W., Kelley, E. \& Stall, J., 1978. Historical and current heavy metal residues in Hudson river fish. - Bull. environ. Contam. Toxicol. 19, 335-339.

Renfro, W. C., 1972. Radioecology of Zinc-65 in Alder Slough, an arm of the Columbia River. In: The Columbia River Estuary and adjacent ocean waters. Ed. by A. T. Pruter \& D. L. Alverson. Univ. Washington Press, Seattle, 755-776.

Rice, T. R., Baptist, J. P., Cross, F. A. \& Duke, T. W., 1972. Potential hazards from radioactive pollution of the estuary. In: Marine pollution and sea life. Ed. by M. Ruivo. Fishing News Books, London, 272-276.

Steele, J. H., Mc Intyre, A. D., Johnston, R., Baxter, I. G., Topping, G. \& Dooley, H. D., 1973. Pollution studies in the Clyde sea area. - Mar. Pollut. Bull. 4, 153-157.

Ting, R. T., 1973. Distribution of $\mathrm{Zn}, \mathrm{Fe}, \mathrm{Mn}$ and $\mathrm{Sr}$ in marine fishes of different habits. In: Radionuclides in ecosystems. Ed. by D. J. Nelson. U. S. A. E. C., Oak Ridge, Tenn., $709-720$.

Wharfe, J. R. \& van den Broek, L. F., 1977. Heavy metals in macroinvertebrates and fish from the lower Medway estuary, Kent. - Mar. Pollut, Bull. 8, 31-34.

Wright, D. A., 1976. Heavy metals in animals from the North East Coast. - Mar. Pollut. Bull. 7 , $36-38$. 